\title{
Comparaison des races bovines Charolaise, Limousine et Maine-Anjou en race pure et en intercroisement 2. Performances d'engraissement des taurillons purs et F1
}

\author{
B. BONAÏTI, B. BIBÉ *, A. HAVY ${ }^{* *}$ et F. MÉNISSIER \\ avec la collaboration technique de \\ P. Gillard, J.J. Chambeyron, Gisèle Le Métayer \\ et de tout le personnel de l'Unité expérimentale de La Minière
}

Institut National de la Recherche Agronomique, Station de Génétique Quantitative et Appliquée,

Centre de Recherches de Jouy-en-Josas, 78350 Jouy-en-Josas, France

* Institut National de la Recherche Agronomique, Station d'Amélioration Génétique des Animaux, Centre de Recherches de Toulouse, B.P. 27, 31326 Castanet-Tolosan, France

${ }^{* *}$ Adresse actuelle : Institut Technique de l'Elevage Bovin, 149, rue de Bercy, 75012 Paris, France

\section{Résumé}

Cette étude concerne les performances d'engraissement de 231 taurillons issus d'un schéma de croisement diallèle entre les races Charolaise, Limousine et Maine-Anjou ainsi que de 26 taurillons de race Hereford. Des contrôles de consommation et de croissance sont réalisés entre l'âge de 9 mois et l'abattage à 15 ou 18 mois. Les animaux reçoivent ad libitum un régime composé de luzerne déshydratée $(70 \%)$ et de pulpe de betterave $(30 \%)$. Entre 9 et 15 mois, les taurillons Charolais ont une croissance supérieure de $155 \mathrm{~g} /$ jour à celle des Limousins et inférieure de $108 \mathrm{~g} /$ jour à celle des Maine-Anjou. Entre 15 et 18 mois, les écarts de croissance entre races pures sont pratiquement nuls. Les différences de quantités d'aliments ingérés sont plus importantes (13\% entre les races Limousine et Charolaise, $15 \%$ entre la Charolaise et la Maine-Anjou entre 9 et 15 mois). Après 15 mois, la Charolaise se rapproche de la Limousine et s'écarte de la MaineAnjou. Vis-à-vis de l'efficacité alimentaire, la Limousine est supérieure à la Charolaise, elle-même supérieure à la Maine-Anjou. Les écarts sont faibles entre 9 et 15 mois $(2,9 \%$ entre les races Limousine et Charolaise, 6,5\% entre la Charolaise et la Maine-Anjou) mais importants dans la seconde période d'engraissement. L'avantage de la race Limousine est alors de $9 \%$ sur la Charolaise et de $30 \%$ sur la Maine-Anjou. La race Hereford a une consommation proche de celle de la Limousine et une efficacité alimentaire proche de celle de la Maine-Anjou pour les deux périodes d'engraissement. Entre 9 et 15 mois, l'effet d'hétérosis est de 3,3\% sur la vitesse de croissance et de $1,5 \%$ sur l'efficacité alimentaire. Il est négatif après 15 mois.

Mots clés : bovins, production de viande, croisement, comparaison de races, hétérosis, Charolaise, Limousine. Maine-Anjou, Hereford, vitesse de croissance, efficacité alimentaire, capacité d'ingestion.

\section{Summary \\ Comparison between Charolais, Limousin and Maine-Anjou breeds in pure- and cross-breeding \\ 2. Fattening results of purebred and F1 young bulls}

A total of 231 young bulls produced by a diallel cross between Charolais, Limousin and Maine-Anjou breeds as well as 26 Hereford purebreds were fattened from 9 to 15 or 18 months. They were fed ad libitum with dehydrated alfalfa $(70 \%)$ and beet root pulp (30\%). Between 9 
and 15 months, Charolais had growth rate $155 \mathrm{~g} / \mathrm{d}$ higher than Limousin and $108 \mathrm{~g} / \mathrm{d}$ lower than Maine-Anjou. Hereford growth rate was $52 \mathrm{~g} / \mathrm{d}$ lower than Limousin. From 15 to 18 months, differences in growth rate were negligible among Limousin, Charolais and Maine-Anjou but all were 202 to $232 \mathrm{~g} / \mathrm{d}$ above Hereford. Daily feed intake differences were higher $(13 \%$ between Limousin and Charolais, $15 \%$ between Charolais and Maine-Anjou from 9 to 15 months). After 15 months, Charolais was nearer to Limousin and farther from Maine-Anjou. Limousin exhibited higher feed efficiency than Charolais, which was better than Maine-Anjou. Feed efficiency differences were small between 9 and 15 months $(2.9 \%$ between Limousin and Charolais, $6.5 \%$ between Charolais and Maine-Anjou) but larger during the second fattening period. Then, the Limousin's advantage was $9 \%$ over Charolais and $30 \%$ over Maine-Anjou. During the two fattening periods, Hereford was close to Limousin for feed intake and to Maine-Anjou for feed efficiency. Between 9 and 15 months, heterosis effect was $3.3 \%$ for growth rate and $1.5 \%$ for feed efficiency. Heterosis in these two traits was negative after 15 months.

Key words : beef cattle, crossbreeding, breed comparison, heterosis, Charolais, Limousin, Maine-Anjou, Hereford, growth rate, feed efficiency, feed intake.

\section{Introduction}

Une expérience de croisement entre trois races à viande françaises, Charolaise, Limousine et Maine-Anjou, fut réalisée en France à partir de 1970 pour analyser la variabilité et estimer les paramètres génétiques du croisement entre ces races pour les différents caractères zootechniques qui intéressent la production de viande à partir des troupeaux de vaches allaitantes (Ménissier et al., 1988). Cette étude concerne plus particulièrement l'aptitude à produire de la viande dans le cadre d'une production de taurillons engraissés intensivement : vitesse de croissance, quantités d'aliments ingérés et efficacité alimentaire. Elle donne une comparaison des trois races à viande françaises et de la Hereford ainsi qu'une estimation des effets d'hétérosis directs.

\section{Matériel et méthodes}

\section{A. Matériel animal}

Les taurillons utilisés proviennent du plan diallèle de croisement entre les trois races Charolaise, Limousine et Maine-Anjou et des accouplements en race pure Hereford (phases I.1 et I.2 décrites par MéNISSIER et al., 1988). Les veaux sont nés au printemps entre 1972 et 1976 et, pour la majorité d'entre eux, sur le domaine expérimental INRA de Galle (situé près de Bourges). En 1976, une partie des veaux issus du même troupeau parental est née dans un autre domaine expérimental INRA (Le Vieux Pin) en raison du transfert de leurs mères pour une nouvelle étape expérimentale (Ménissier et al., 1988). Tous les veaux mâles vivants après le sevrage sont concernés par cette étude des performances d'engraissement.

\section{B. Conduite alimentaire et protocole de contrôle durant l'engraissement}

Les conditions d'alimentation sont restées homogènes durant toute l'expérience. Les veaux restent en permanence avec leur mère jusqu'au sevrage à l'âge constant de 6 mois et demi. A partir de l'âge de deux mois, ils peuvent consommer du foin de bonne 
qualité et une quantité d'aliments concentrés limitée à $1,2 \mathrm{~kg}$ par jour. Les poids à 180 jours des veaux Charolais, Limousins et Maine-Anjou sont respectivement de 236, 215 et $248 \mathrm{~kg}$, les croisés présentent un. hétérosis de $5 \mathrm{~kg}$ et les Hereford n'atteignent que $187 \mathrm{~kg}$ (QuesNel, 1980). Après le sevrage, ils disposent d'une alimentation à base de foin et de concentrés. A l'âge de huit mois, les veaux sont transférés dans une unité expérimentale d'engraissement où ils restent attachés en stalle longue sur une litière de paille (INRA-La Minière). Le protocole de contrôle de l'engraissement commence à l'âge de neuf mois après une période de transition de un mois. La ration distribuée sous forme de pellets est composée de $70 \%$ de luzerne et de $30 \%$ de pulpes de betterave déshydratées $(91 \%$ de matière sèche, $0,69 \mathrm{UFV}$ et $100 \mathrm{~g}$ de PDI par $\mathrm{kg}$ de matière sèche). Cette ration était complétée par un condiment minéral vitaminé (100 à $150 \mathrm{~g} / \mathrm{j}$ ). Pour assurer une alimentation ad libitum, les refus étaient mesurés quatre fois par semaine et à cette occasion les quantités distribuées étaient, après une ration initiale de $6,5 \mathrm{~kg}$, augmentées ou diminuées de $0,5 \mathrm{~kg}$ si la quantité de refus observée était respectivement inférieure à $1 \mathrm{~kg}$ ou supérieure à $2 \mathrm{~kg}$. De faibles quantités de paille, non contrôlées, sont consommées par les animaux à partir de leur litière. Les animaux sont pesés tous les mois. Ils sont regroupés par lot d'engraissement selon leur date de naissance. A l'intérieur d'un lot, les différences d'âge en début de contrôle n'excèdent pas trois semaines. A partir de neuf mois, la durée de l'engraissement était de 182 jours pour une moitié des taurillons ou de 294 jours pour l'autre moitié. L'affectation des animaux à chacun de ces deux âges d'abattage est faite chaque année de façon aléatoire pour chacun des 10 types génétiques. Les effectifs sont donnés selon le génotype et l'âge en fin d'engraissement dans le tableau 1.

\section{TABLEAU 1}

Effectifs des animaux engraissés selon le type génétique

Numbers of fattened bulls according to genetic type

\begin{tabular}{|c|c|c|c|c|}
\hline \multirow{2}{*}{ Race paternelle } & \multicolumn{4}{|c|}{ Race maternelle } \\
\hline & Limousine & Charolaise & Maine-Anjou & Hereford \\
\hline Limousine & $21-11 *$ & $20-10$ & $22-11$ & \\
\hline Charolaise . . . . . . . . . & $34-17$ & $30-15$ & $24-8$ & \\
\hline$\ldots \ldots \ldots \ldots$ & $29-16$ & $30-13$ & $21-9$ & \\
\hline Hereford . . . . . . . . & & & & $26-13$ \\
\hline
\end{tabular}

* Le premier nombre donne l'effectif total des animaux engraissés, le second celui de ceux engraissés jusqu'à 18 mois. The first figure gives the total number of fattened bulls, the second gives the number of those fattened up to 18 months.

\section{Variables étudiées}

Les variables suivantes sont analysées : poids au début du contrôle (273 jours d'âge), à 182 jours et à 294 jours d'engraissement (respectivement 15 et 18 mois d'âge), vitesse de croissance ( $\mathrm{g} / \mathrm{jour})$, consommation journalière d'aliment $(\mathrm{kg} / \mathrm{jour})$, efficacité alimentaire (vitesse de croissance/consommation journalière en $\mathrm{g} / \mathrm{kg}$ ), appétit (consommation par $100 \mathrm{~kg}$ de poids vif moyen) pour chacune des trois périodes, 9-15, 15-18 et 9-18 mois. 


\section{Méthodes d'analyse}

L'analyse des données est réalisée par la méthode des moindres carrés selon un modèle faisant intervenir la régression sur l'âge en début de contrôle et quatre effets additifs : le type génétique (10 génotypes correspondant aux 4 races pures et aux 6 croisements $\mathrm{F} 1$ ), le lot d'engraissement (18 lots répartis sur 5 années) et le lieu de naissance (Galle pour les 4 premières années, Galle ou le Vieux Pin pour la dernière année).

Des combinaisons linéaires des effets «type génétique " sont calculées pour obtenir les effets d'hétérosis directs $\left(h^{I}\right)$ et les effets propres à chaque croisement $\mathrm{F} 1$ (moyenne des deux croisements réciproques) (Ménissier et al., 1988). L'estimation des effets maternels à partir de ces données n'est pas donnée en raison de leur faible valeur espérée vis-à-vis du biais lié aux différences de niveaux génétiques entre les parents mâles et femelles (MéNissier et al., 1988). Les variances d'erreur de ces combinaisons linéaires sont obtenues en utilisant la matrice de variance et covariance entre estimées. Les tests de signification de ces différences ne tiennent compte que du risque d'erreur propre à la seule comparaison élémentaire concernée.

\section{Résultats}

Les tests de signification pour chacun des facteurs de variation sont présentés dans le tableau 2. Les estimées des types génétiques et des effets d'hétérosis sont données dans les tableaux 3 et 4 .

\section{A. Les effets de milieu}

L'effet lot est significatif pour de nombreuses variables, en particulier pour les poids à 9,15 et 18 mois. Les différences résultent entre autres des conditions climatiques, qui ont joué surtout sur la croissance des veaux avant le sevrage, et de l'évolution de l'âge des mères. L'utilisation exclusive, en vue du premier vêlage à deux ans en 1972, de deux pères à plus faible poids de naissance pour chaque race paternelle (MÉnissier et al., 1988) explique aussi pour une part les moindres performances obtenues durant la première année. C'est en 1974 que le poids est le plus élevé. Sauf pour la première année, les différences initiales de poids sont en partie compensées par une meilleure vitesse de croissance en engraissement grâce à une consommation et une efficacité alimentaires plus élevées.

Les animaux nés au Vieux Pin en 1976 ont, en début de contrôle, un poids inférieur de $30 \mathrm{~kg}$ à celui de ceux nés à Galle. Ceci résulte du déficit d'herbe paturée, lié à la sécheresse particulièrement importante de 1976, et qui n'a pas été suffisamment compensé par des apports de fourrages.

L'effet de l'âge sur le poids en début d'engraissement ( 9 mois \pm 20 jours) est significatif et traduit la croissance avant l'entrée en station. Les animaux plus âgés ont aussi, de façon significative. un appétit et une efficacité alimentaire plus faibles. 


\section{TABLeau 2}

Analyse de variance

Analysis of variance

\begin{tabular}{|c|c|c|c|c|c|c|c|}
\hline \multirow{2}{*}{$\begin{array}{l}\text { Facteur de variation } \\
\text { Variables }\end{array}$} & \multicolumn{2}{|c|}{ Résiduelle } & \multirow{2}{*}{$\begin{array}{c}\begin{array}{c}\text { Lot } \\
\text { d'engrais- } \\
\text { sement }\end{array} \\
F\end{array}$} & \multirow{2}{*}{$\begin{array}{c}\begin{array}{c}\text { Lieu de } \\
\text { naissance }\end{array} \\
F\end{array}$} & \multirow{2}{*}{$\begin{array}{c}\begin{array}{c}\text { Types gé- } \\
\text { nétiques }\end{array} \\
F\end{array}$} & \multirow{2}{*}{$\begin{array}{c}\begin{array}{c}\text { Age au } \\
\text { début }\end{array} \\
F\end{array}$} & \multirow[t]{2}{*}{$\begin{array}{c}R^{2} \\
(\%)\end{array}$} \\
\hline & $\sigma$ & $\mathrm{CV}$ & & & & & \\
\hline Poids à : $\begin{array}{r}9 \text { mois }(\mathrm{kg}) \\
15 \text { mois }(\mathrm{kg}) \\
18 \text { mois }(\mathrm{kg})\end{array}$ & $\begin{array}{l}29 \\
43 \\
46\end{array}$ & $\begin{array}{r}10,2 \\
7,9 \\
6,8\end{array}$ & $\begin{array}{l}* * \\
* * \\
* *\end{array}$ & $\begin{array}{l}* * \\
*\end{array}$ & $\begin{array}{l}* * \\
* * \\
* *\end{array}$ & $* *$ & $\begin{array}{l}56 \\
54 \\
63\end{array}$ \\
\hline $\begin{array}{ll}\text { de } 9 \text { à } 18 \text { mois : } \\
\text { VC } \quad(g / \text { jour }) \ldots \ldots \\
\text { Cons. } & (\mathrm{kg} / \mathrm{jour}) \ldots \ldots \\
\text { EA } & (\mathrm{g} / \mathrm{kg}) \ldots \ldots \ldots \\
\text { App. } & (\mathrm{kg} / 100 \mathrm{~kg}) \ldots \ldots\end{array}$ & $\begin{array}{l}117 \\
1,0 \\
9 \\
0,13\end{array}$ & $\begin{array}{l}9,0 \\
9,2 \\
7,3 \\
5,8\end{array}$ & $\begin{array}{l}* \\
* *\end{array}$ & $*$ & $\begin{array}{l}* * \\
* * \\
* * \\
* *\end{array}$ & $* *$ & $\begin{array}{l}54 \\
53 \\
54 \\
56\end{array}$ \\
\hline $\begin{array}{ll}\text { de } 9 \text { à } 15 \text { mois : } \\
\text { VC } \quad(g / \text { jour }) \ldots \ldots \\
\text { Cons. } & (\mathrm{kg} / \text { jour }) \ldots \ldots \\
\text { EA } & (\mathrm{g} / \mathrm{kg}) \ldots \ldots \\
\text { App. } & (\mathrm{kg} / 100 \mathrm{~kg}) \ldots\end{array}$ & $\begin{array}{l}164 \\
1,0 \\
13 \\
0,16\end{array}$ & $\begin{array}{r}11,6 \\
9,7 \\
9,7 \\
6,4\end{array}$ & $\begin{array}{l}+ \\
+ \\
+ \\
* \\
* *\end{array}$ & & $\begin{array}{l}* * \\
* * \\
* * \\
* *\end{array}$ & $\begin{array}{l}* \\
* \\
* *\end{array}$ & $\begin{array}{l}36 \\
47 \\
29 \\
43\end{array}$ \\
\hline $\begin{array}{ll}\text { de } 15 \text { à } 18 \text { mois : } \\
\text { VC } \quad(g / \text { jour }) \ldots \ldots \\
\text { Cons. } \quad(\mathrm{kg} / \text { jour }) \ldots \ldots \\
\text { EA } \quad(\mathrm{g} / \mathrm{kg}) \ldots \ldots \\
\text { App. } \quad(\mathrm{kg} / 100 \mathrm{~kg}) \ldots\end{array}$ & $\begin{array}{c}177 \\
1,2 \\
14 \\
0,14\end{array}$ & $\begin{array}{r}16,7 \\
10,6 \\
15,4 \\
7,6\end{array}$ & $\begin{array}{l}* * \\
+ \\
+\end{array}$ & & $\begin{array}{l}* \\
* * \\
* * \\
* *\end{array}$ & & $\begin{array}{l}45 \\
44 \\
43 \\
45\end{array}$ \\
\hline $\begin{array}{ll}\sigma & : \text { écart-type résiduel } \\
\mathrm{CV} & : \text { coefficient de varia } \\
F & : \text { test de Fisher sigr } \\
& +: P<0.10 . \\
R^{2} & : \text { pourcentage de la } \\
\mathrm{VC} & : \text { vitesse de croissan } \\
\text { Cons. : consommation jour } \\
\mathrm{EA} & \text { : efficacité alimentai } \\
\text { App. } & \text { : appétit ; appetite ( }\end{array}$ & $\begin{array}{l}\text { residual } \\
\text { ion ; coef } \\
\text { ficatif au } \\
\text { ariance e } \\
; \text { growth } \\
\text { alière ; } d \\
\text {; feed ef } \\
\text { iily feed }\end{array}$ & $\begin{array}{l}\text { tandard } \\
\text { ficient o } \\
\text { niveau } \\
\text { spliqué } \\
\text { rate. } \\
\text { aily feed } \\
\text { ficiency. } \\
\text { onsump }\end{array}$ & $\begin{array}{l}\text { deviation. } \\
\text { f variation. } \\
\text {; Fisher's te. } \\
\text { par le modèl } \\
\text { t consumptio } \\
\text { tion / mean }\end{array}$ & $\begin{array}{l}\text { t significant } \\
\text {; R. Squart } \\
\text { eight). }\end{array}$ & $\begin{array}{l}\text { at level: }{ }^{* *} \\
d \text { of the moc }\end{array}$ & $P<0.01$ & $P<0.05$ \\
\hline
\end{tabular}

\section{B. Les effets génétiques}

Les différences entre types génétiques sont significatives pour toutes les variables (tableaux 3 et 4 ).

\section{Comparaison entre races pures}

Les quatre races pures se classent pour les poids aux différents âges en ordre croissant de la façon suivante : Hereford, Limousine, Charolaise, Maine-Anjou. La 


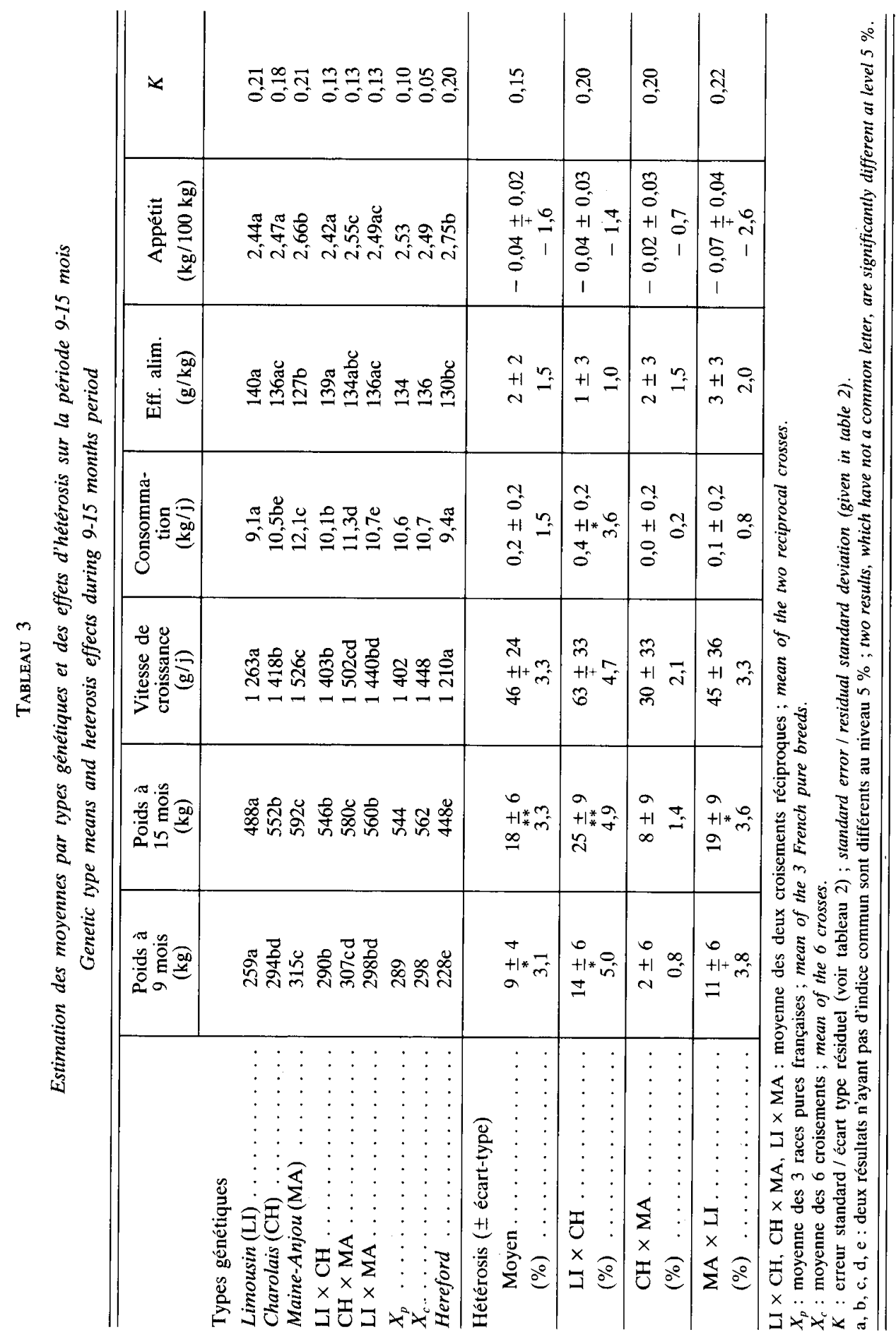




\begin{tabular}{|c|c|c|c|c|c|c|c|c|c|}
\hline & & $x$ & 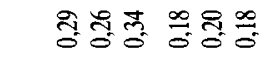 & 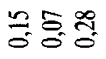 & $\overline{\tilde{\delta}}$ & స్రి & $\stackrel{\infty}{\sigma}$ & $\overrightarrow{\tilde{\sigma}}$ & \\
\hline & & 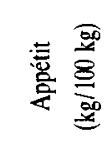 & 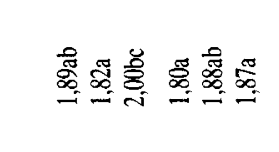 & gू & 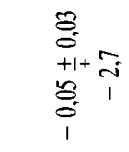 & $\begin{array}{ll}\mathbb{g} & \\
0 & \\
+1 & 0 \\
0 & 1 \\
0 & 1 \\
0 & 1 \\
1 & \end{array}$ & $\begin{array}{l}2 \\
0 \\
0 \\
+1 \\
+1 \\
0 \\
0 \\
0 \\
1\end{array}$ & $\begin{array}{ll}2 & \\
0 & \\
+1 & = \\
0 & 0 \\
0 & 1 \\
1 & 1\end{array}$ & \\
\hline 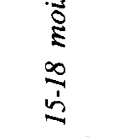 & 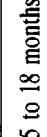 & 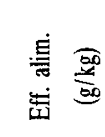 & 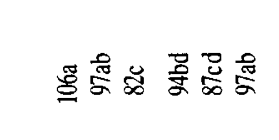 & ஃ๙ & 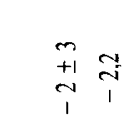 & $\begin{array}{l}+ \\
+1 * g \\
i \\
1\end{array}$ & $\begin{array}{ll}n & 0 \\
+1 & \sim \\
i & 1 \\
1 & 1\end{array}$ & $\stackrel{n}{+1} \underset{m}{m}$ & \\
\hline 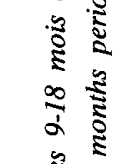 & 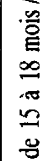 & $\begin{array}{l}\text { 亶 } \\
\text { 兽 }\end{array}$ & 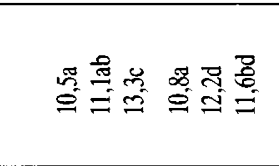 & $\stackrel{0}{=} \stackrel{\circ}{=} \stackrel{8}{=}$ & 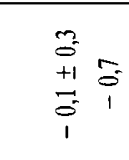 & $\begin{array}{l}\stackrel{0}{0} \\
+1 \\
0 \\
8\end{array}$ & 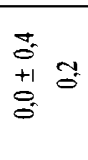 & 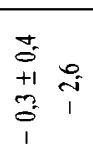 & \\
\hline 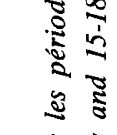 & & 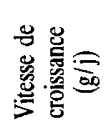 & 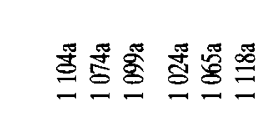 & ริ용 & 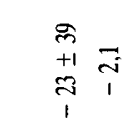 & $\begin{array}{cc}\bar{n} & 9 \\
+1 & \text { n } \\
3 & 1 \\
1 & \end{array}$ & $\begin{array}{c}\infty \\
+1 \\
\stackrel{+1}{N}\end{array}$ & $\stackrel{n}{+1}=$ & \\
\hline 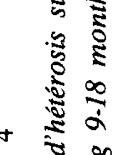 & & 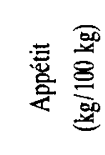 & 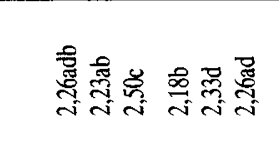 & 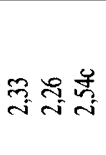 & 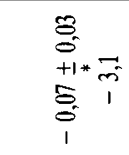 & 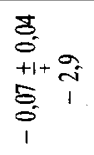 & 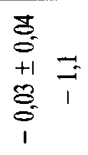 & 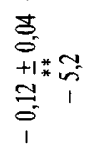 & \\
\hline 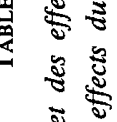 & 美 & $\begin{array}{l}\text { 禀 } \\
\text { 㟢 }\end{array}$ & 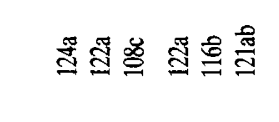 & $\cong \cong$ & $\underset{n}{+1}=$ & $\overbrace{1}^{m} \tilde{0}$ & $\stackrel{m}{+1}=$ & $\stackrel{m}{+1+m}$ & \\
\hline 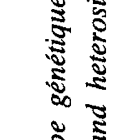 & 荥 & 蜜 & 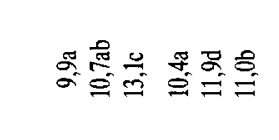 & E兽 & 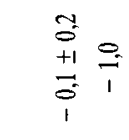 & $\begin{array}{l}3 \\
0 \\
+1 \\
0 \\
0\end{array}$ & $\begin{array}{l}m \\
0 \\
+1 \\
8 \\
8\end{array}$ & 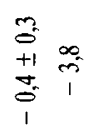 & \\
\hline 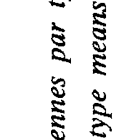 & $\approx$ & 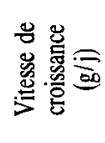 & 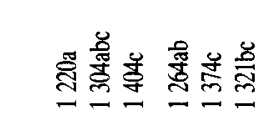 & 함 쥼융 & 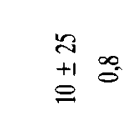 & $\stackrel{+}{+1}=$ & $\begin{array}{l}\infty \\
+1 \\
\approx\end{array}$ & $\stackrel{5}{+1}=$ & $\dot{m}$ \\
\hline 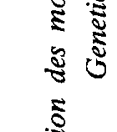 & & 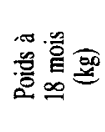 & 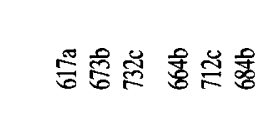 & 运运 & $\begin{array}{l}0 \\
+1 \\
0\end{array}=$ & $\stackrel{m}{+1} 9$ & $\stackrel{\sim}{+1} \underset{=}{=}$ & $\stackrel{n}{+1} \underset{a}{a}=$ & 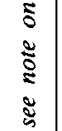 \\
\hline 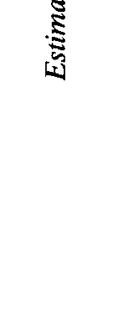 & & & 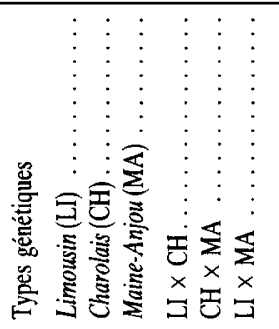 & 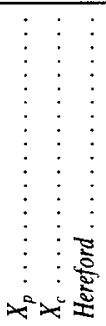 & 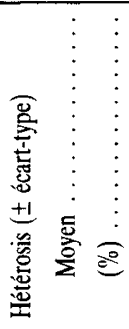 & 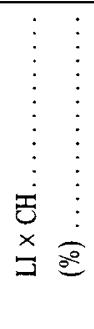 & 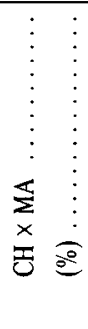 & 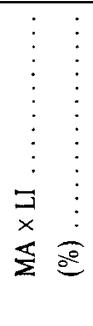 & 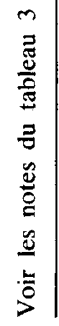 \\
\hline
\end{tabular}


différence entre la Charolaise et la Maine-Anjou se maintient entre 7 et $9 \%$ en valeur relative pour les poids à 9,15 et 18 mois. La race Limousine, qui marque un retard de poids de $12 \%$ sur la Charolaise à 9 et 15 mois, comble en partie cette différence à 18 mois ( $8 \%$ d'écart). L'avantage de la Limousine sur la Hereford décroît de 12 à $8 \%$ entre 9 et 15 mois et remonte à $11 \%$ pour le poids à 18 mois.

Les différences de vitesse de croissance correspondent à celles observées pour les poids. Entre 9 et 15 mois, la race Charolaise a une croissance supérieure de $155 \mathrm{~g} / \mathrm{jour}$ à celle de la Limousine et inférieure de $108 \mathrm{~g} /$ jour à celle de la Maine-Anjou. Les écarts de croissance sont pratiquement nuls entre 15 et 18 mois. La croissance de la Hereford se maintient à un niveau proche de la Limousine entre 9 et 15 mois $(-52 \mathrm{~g} /$ jour $)$ mais devient très inférieure après 15 mois $(-232 \mathrm{~g} /$ jour $)$.

Le classement des trois races françaises pour la consommation quotidienne correspond à celui donné pour la vitesse de croissance. Entre 9 et 15 mois, les différences de consommation sont plus importantes (13\% entre la Limousine et la Charolaise, $15 \%$ entre la Charolaise et la Maine-Anjou) et conduisent, pour l'efficacité alimentaire, à des différences opposées mais plus faibles en valeur relative. Le classement des races est vis-à-vis de l'efficacité alimentaire : Maine-Anjou, Charolaise, Limousine, par ordre croissant. Pour ce dernier critère, la Charolaise est plus proche de la Limousine $(-2,9 \%$ en valeur relative) que de la Maine-Anjou (+ 6,5\%). Exprimées en terme d'appétit (consommation par $100 \mathrm{~kg}$ de poids vif), les différences de consommation sont plus faibles mais de même signe. Pour ce critère aussi, la Charolaise est plus proche de la Limousine que de la Maine-Anjou. La Hereford a une consommation supérieure à la Limousine et un plus grand appétit que les trois races françaises. Cette consommation plus importante conduit à une efficacité alimentaire défavorable de la Hereford quoique légèrement supérieure à celle de la Maine-Anjou, la moins bonne des races françaises pour ce critère.

Entre 15 et 18 mois, on retrouve entre la Limousine et la Maine-Anjou un écart de consommation légèrement inférieur à ce qui est observé sur la période précédente ( $25 \%$ au lieu de $29 \%$ en valeur relative). En revanche, la consommation de la Charolaise se rapproche très nettement de la Limousine $(+5 \%)$ et s'écarte notablement de la Maine-Anjou (- $20 \%)$. L'appétit de la Charolaise est cependant le plus faible des trois races françaises : $1,82-1,89$ et $2,00 \mathrm{~kg}$ d'aliment pour $100 \mathrm{~kg}$ de poids vif respectivement pour les trois races Charolaise, Limousine et Maine-Anjou. La réduction des écarts de vitesse de croissance associée à un maintien des écarts de consommation conduit à une augmentation très importante des différences d'efficacité alimentaire entre les trois races françaises; l'avantage de la Limousine est durant la période 15-18 mois de $9 \%$ sur la Charolaise et de $30 \%$ sur la Maine-Anjou.

La consommation de la race Hereford entre 15 et 18 mois est très voisine de celle de la Limousine; malgré ceci et à cause d'une forte réduction de la vitesse de croissance, son efficacité alimentaire reste faible et très proche de celle de la MaineAnjou.

Sur la durée totale de l'engraissement de 9 à 18 mois, on retrouve des résultats similaires à ceux obtenus entre 9 et 15 mois. La supériorité de la Maine-Anjou sur la Charolaise est de $8 \%$ pour la vitesse de croissance, de $22 \%$ pour la consommation et de $12 \%$ pour l'appétit. Ces écarts, qui sont plus marqués que ne le laisse espérer la combinaison des résultats obtenus sur les deux périodes, résultent en partie du fait que les taurillons Maine-Anjou retenus pour l'abattage à 18 mois avaient en moyenne une consommation et une vitesse de croissance légèrement supérieure à ceux retenus pour 
l'abattage à 15 mois. L'écart de $11,6 \%$ observé entre les deux races pour l'efficacité alimentaire est cohérent avec la combinaison des résultats sur les deux périodes élémentaires. La Limousine a une consommation et une vitesse de croissance respectivement inférieures de 7,5 et de $6 \%$ à la Charolaise. Pour l'efficacité alimentaire, la Limousine ne marque qu'un léger avantage de $1 \%$ alors qu'elle avait un avantage de 2,4 et $8 \%$ sur les deux périodes d'engraissement. La race Hereford a, sur l'ensemble de l'engraissement, une croissance inférieure de $130 \mathrm{~g}$ à la Limousine, tout en ayant la même consommation d'aliments. Son efficacité alimentaire est voisine de la MaineAnjou.

\section{Les effets d'hétérosis}

On observe un hétérosis moyen de $3 \%$ en valeur relative pour les poids à 9 et 15 mois. Cet avantage se réduit à $1,9 \%$ pour le poids à 18 mois. Seuls les effets obtenus à 9 et 15 mois sont significatifs. Entre types de croisement, il existe une certaine hétérogénéité des effets d'hétérosis spécifique. Le croisement entre les races Limousine et Charolaise donne un effet relativement élevé (5\% à 9 ou 15 mois) alors que les valeurs d'hétérosis entre les races Charolaise et Maine-Anjou ne sont que de 0,8 et $1,4 \%$ respectivement pour les poids à 9 et 15 mois. Le croisement Maine-Anjou $\times$ Limousine est intermédiaire entre ces deux extrêmes. Il est à souligner que ces valeurs d'hétérosis spécifique ne sont pas significativement différentes entre elles.

L'hétérosis sur la vitesse de croissance entre 9 et 15 mois est de 3,3\% mais il n'est significatif qu'au niveau $10 \%$. Comme on observe aussi un effet d'hétérosis sur la consommation de $1,5 \%$, l'hétérosis sur l'efficacité alimentaire n'est que de 1,5\%. Ces deux derniers effets ne sont pas significatifs. L'avantage des taurillons croisés pour la consommation est un avantage lié à leur poids vif plus élevé car l'hétérosis sur l'appétit est négatif $(-1,6 \%)$. On observe encore pour la vitesse de croissance et la consommation d'aliments la même variabilité d'hétérosis entre croisements, mais les différences entre effets d'hétérosis spécifique ne sont pas significatives.

Entre 15 et 18 mois, l'hétérosis sur la vitesse de croissance est négatif mais non significatif. Ce désavantage des taurillons croisés s'accompagne d'une réduction de l'efficacité alimentaire $(-2,2 \%)$. L'appétit présente un effet d'hétérosis négatif de $-2,7 \%$. Cette inversion des effets d'hétérosis entre les deux périodes (9-15 et 15-18 mois) est plus élevée pour les croisés Charolais $\times$ Limousins qui présentaient avant 15 mois l'hétérosis spécifique le plus élevé.

Sur l'ensemble de la période de 9 à 18 mois, les effets d'hétérosis sont relativement faibles et non significatifs : $0,8 \%$ sur la vitesse de croissance et $1,5 \%$ sur l'efficacité alimentaire. Seul l'hétérosis sur l'appétit est significatif mais négatif : $-3,1 \%$.

\section{Discussion}

Il ressort clairement de nos résultats que pour la vitesse de croissance des taurillons entre 9 et 15 mois, la race Maine-Anjou est supérieure à la Charolaise de $7 \%$ en valeur relative, elle-même supérieure à la Limousine de $11 \%$. En croisement sur un troupeau laitier (ANDERSEn et al., 1977 ; EVERITT et al., 1980 ; MÉNISSIER et al., 1982 ; 
RENAND, 1985) ou sur un troupeau de vaches allaitantes (BASs et al., 1981 ; Gregory et al., 1982 ; RAHNEFELD et al., 1983) les résultats concernant le poids en fin d'engraissement par jour d'âge se situent entre -5 et $-10 \%$ pour l'écart entre les races Limousine et Charolaise avec une moyenne de $-7 \%$. Notre résultat est donc cohérent avec ce dernier chiffre, bien que plus faible si on admet qu'en croisement on n'obtient que la demi-différence des races pures. Cet écart de croissance reflète aussi les écarts de poids habituellement observés pour les vaches adultes Charolaises et Limousines.

La supériorité de croissance de la Maine-Anjou qui résulte de cette comparaison n'a été observée jusqu' à présent que par Bass et al. (1981) dans une expérience de croisement sur mère Angus et de façon beaucoup plus faible. Dans deux larges expériences de comparaison de races rapportées par GREGORY et al. (1982) et MéNISSIER et al. (1982), les croisés Charolais permettent au contraire des croissances après sevrage plus importantes que les Maine-Anjou. La supériorité de la Charolaise apparaît aussi à travers les poids adultes observés sur le troupeau des mères à Galle $(712 \mathrm{~kg}$ au lieu de $648 \mathrm{~kg}$ d'après Villareal, 1985). Cependant, dans cette situation, les vaches MaineAnjou ont une production laitière beaucoup plus élevée que les Charolaises et ne parviennent peut-être pas à exprimer au stade adulte leur potentiel de croissance de façon aussi complète. L'infériorité de croissance des taurillons Hereford vis-à-vis des Charolais, proche de $14 \%$, est légèrement inférieure au double de la moyenne de $9 \%$ des écarts obtenus en croisement par CARTER (1975), Everitr et al. (1980), Gregory et al. (1982) ainsi que MÉnISSIER et al. (1982). Pour la vitesse de croissance entre 9 et 15 mois, on retrouve donc en grande partie les données bibliographiques déjà publiées. L'avantage de la Charolaise est moins marqué dans nos résultats. En revanche, la Maine-Anjou apporte un potentiel de croissance supérieur aux deux autres races françaises. Pour la période 15-18 mois, il n'apparaît en revanche aucune différence de croissance entre ces trois races. Il est possible que cette réduction des écarts soit en partie le résultat des conditions de stabulation entravée qui sont plus dommageables aux animaux de grande taille : trois taurillons croisés Charolais $\times$ Maine-Anjou n'ont pas pu terminer l'engraissement jusqu'à 18 mois en raison des problèmes de pattes ou d'aplombs alors qu'aucun problème n'a été rencontré avec ceux de petite taille, les Limousins en particulier. Avec un mode d'utilisation complètement différent, des veaux mâles issus du même troupeau maternel (production de bœufs à l'herbe jusqu'à 30 mois avec un engraissement terminal à l'auge de 2 mois), il est intéressant de noter que le potentiel de croissance de la race Charolaise est mieux mis en valeur puisque cette race obtient entre le sevrage et l'abattage une vitesse de croissance supérieure de $14,5 \%$ en valeur relative à la Limousine et très proche de la Maine-Anjou (Frebling et al., 1982 ; GillaRd et al., 1986).

Entre 9 et 15 mois, les différences d'efficacité alimentaire observées dans cette étude sont plus faibles que celles observées pour la vitesse de croissance. Il est constaté aussi que l'augmentation de la vitesse de croissance est accompagnée d'une détérioration de l'efficacité alimentaire. En revanche, l'efficacité alimentaire augmente lorsque la proportion de tissu adipeux dans le gain de poids diminue : les trois races Limousine, Charolaise et Maine-Anjou ont à 15 mois respectivement $12,0,12,4$ et $14,5 \%$ de gras dans la carcasse (BonAïTI et al., 1988). La composition joue donc, dans ces conditions d'engraissement et pour cet échantillon de races, un rôle plus important que le niveau de production (besoins d'entretien et de croissance/besoin d'entretien) pour déterminer l'efficacité alimentaire. Entre 15 et 18 mois, les différences de composition de carcasse se sont aggravées puisque les races Charolaise et Maine-Anjou ont respectivement 13,9 et $17,5 \%$ de gras dans la carcasse en fin d'engraissement alors que la proportion de gras de la Limousine reste, comme à 15 mois, égale à $12 \%$ (Bonä̈rı et al., 1988). Ces 
écarts, qui correspondent à des différences plus importantes encore de la proportion des dépôts adipeux dans le gain de poids entre 15 et 18 mois, se traduisent naturellement par une efficacité alimentaire moindre de la Charolaise et surtout de la Maine-Anjou vis-à-vis de la Limousine. Les écarts d'efficacité alimentaire sont plus importants durant la seconde période d'engraissement d'une part parce que les écarts de composition corporelle sont plus élevés et d'autre part parce que ceux-ci ne sont plus compensés par des écarts de niveau de production. Il faut noter, en revanche, que la Hereford, malgré une proportion de gras de carcasse beaucoup plus importante (respectivement 18,1 et $23,7 \%$ à 15 et 18 mois), a une efficacité alimentaire très proche de celle de la MaineAnjou, probablement parce que ses besoins d'entretien sont plus faibles. Cet avantage d'efficacité alimentaire de la race Charolaise et surtout de la Limousine sur la MaineAnjou et la Hereford se distingue des résultats de Gregory et al. (1982), Liboriussen et al. (1982) et de Ménissier et al. (1982) qui n'observent pas de différences aussi importantes et classent la Charolaise devant la Limousine.

L'effet d'hétérosis moyen sur la vitesse de croissance ou sur le poids des taurillons de 9 à 15 mois est faible (environ $3 \%$ ). Il est compris dans l'intervalle de 0 à $5 \%$ où se regroupent la plupart des estimations rassemblées par BiBE \& Foulley (1976) ainsi que par LoNG (1980) et qui correspondent à des croisements entre les races Hereford, Angus, Shorthorn et Charolaise. Cet hétérosis moyen est proche de celui observé sur le poids au sevrage des veaux mâles et femelles du même troupeau expérimental $(2,1 \%$ selon QUESNEL, 1980). En revanche, on observe un hétérosis négatif mais non significatif pour la vitesse de croissance au-delà de 15 mois. Cette évolution de l'hétérosis avec l'âge est relativement cohérente avec les résultats rassemblés par LoNG (1980) qui montre que l'effet d'hétérosis s'annule lorsque les animaux atteignent le stade adulte. Les conditions intensives d'engraissement propre à cette étude sont favorables à une bonne extériorisation de l'hétérosis selon Klosterman et al. (1968, cités par BiBÉ \& Foulley, 1976), LASLEY et al. (1973), LoNG \& Gregory (1975) qui observent un hétérosis plus élevé pour un engraissement intensif comparé à un engraissement semiintensif. Pour les systèmes de production de viande en France, intensif ou à l'herbe, il est donc peu probable de pouvoir obtenir des effets d'hétérosis plus importants que 3 à $5 \%$ sur la vitesse de croissance.

Les estimations de l'hétérosis sur l'efficacité alimentaire sont moins nombreuses que pour la vitesse de croissance. Nos résultats sont en accord avec BıBÉ \& FoulleY (1976) qui observent que les quelques expériences portant sur ce caractère ne permettent pas d'affirmer qu'il existe un effet d'hétérosis marqué. Cependant EllersieK et al. (1977) observent pour l'efficacité alimentaire ajustée pour le poids, un effet d'hétérosis moyen proche de $5 \%$ entre les trois races Charolaise, Angus et Hereford.

Les effets d'hétérosis, qui sont faibles en valeur relative, ne doivent pas être négligés car ils restent importants vis-à-vis des différences entre races pures. Le croisement Limousine $\times$ Charolaise donne ainsi des produits très proches de la race Charolaise tant pour les performances entre 9 et 15 mois que pour les poids à 15 et 18 mois.

\section{Conclusion}

Nos résultats confirment l'important gradient de croissance entre les races Hereford, Limousine, Charolaise et Maine-Anjou (1 210,1263, 1418 et 1526 g/jour entre 9 et 15 mois). Mais ce gradient s'accompagne d'une forte élévation des quantités ingérées 
et on observe pour l'efficacité alimentaire des différences inverses pour les trois races françaises. Entre les trois races, c'est la composition du gain de poids plutôt que le niveau de production qui détermine le classement pour l'efficacité alimentaire. Après 15 mois, on ne relève plus que des différences négligeables pour la vitesse de croissance, mais, en revanche on a, vis-à-vis de l'efficacité alimentaire, un très net avantage des races Limousine et Charolaise sur la Maine-Anjou (106 et $97 \mathrm{~g} / \mathrm{kg}$ vs $82 \mathrm{~g} / \mathrm{kg}$ ). Malgré une consommation proche de celle de la Limousine, la race Hereford n'a qu'une efficacité alimentaire proche de celle de la Maine-Anjou. Comme dans les croisements entre les races Hereford, Angus et Shorthorn, les effets d'hétérosis sur les caractères de croissance sont de l'ordre de $3 \%$. Ils peuvent être plus faibles ou même négatifs pour la vitesse de croissance et l'efficacité alimentaire en fin d'engraissement.

Reçu le 11 juin 1987. Accepté le 13 janvier 1988.

\section{Références bibliographiques}

Andersen B.B., Liboriussen T., Kousgaard K., 1977. Crossbreeding experiment with beef and dual-purpose sire breeds on Danish dairy cows. 3. Daily gain, feed conversion and carcass quality of intensively fed young bulls. Livest. Prod. Sci., 4, 19-29.

Bass J.J., Carter A.H., Johnson D.L., BaKer R.L., Jones K.R., 1981. Sire-breed comparison of carcass composition of steers from Angus dams. J. Agric. Sci., 97, 515-522.

Bibê B., Foulley J.L., 1976. Effet d'hétérosis chez les bovins à viande : Résultats bibliographiques. Bull. Tech. Dép. Génét. Anim., INRA, 24, 87-110.

Bonaïti B., Bibé B., Havy A., Ménissier F., 1988. Comparaison des races bovines Charolaise, Limousine et Maine-Anjou en race pure et en intercroisement. 3. Performances d'abattage des taurillons purs et F1. Génét. Sél. Evol., 20 (sous presse).

Carter A.H., 1975. Evaluation of cattle breeds for beef production in New Zealand. A review. Livest. Prod. Sci., 2, 327-340.

Ellersieck M.R., Lasley J.F., SibBit W.R., Langford L.H., 1977. Heterosis in feed efficiency of beef steers. J. Anim. Sci., 45, 703-707.

Everitt G.C., Jury K.E., Dalton D.C., Langridge M., 1980. Beef production from the dairy herd. 4. Growth and carcass composition of straight-bred and beef-cross Friesian steers in several environments. N. Z. J. Agric. Res., 23, 11-20.

Frebling J., Bonaïri B., Bibé B., Chambeyron J.J., Gillard P., Ménissier F., 1982. Comparisons of fattening and slaughter performances between Charolais, Limousin, Maine-Anjou and Hereford breeds according to various production types. In : $2^{\text {nd }}$ World Congr. Genet. Appl. Livest. Prod., Madrid, 4-8 Oct., 1982, 8, 334-339, Editorial Garsi, Madrid.

Gillard P., Leconte D., Chambeyron J.J., Frebling J., 1986. Production de bœufs de différents types génétiques finis à l'auge à 30 mois (bilan de 7 années). Bull. Tech. C.R.Z.V. Theix, INRA, 65, 31-39.

Gregory K.E., CundifF L.V., КосH R.M., 1982. Comparison in crossbreeding systems and breeding stocks used in suckling herds of continental and temperate areas. In : $2^{\text {nd }}$ World Congr. Genet. Appl. Livest. Prod., Madrid, 4-8 Oct., 1982, 5, 482-503, Editorial Garsi, Madrid.

Lasley J.F., Sibitt B., Lawgford L., Comfort J.E., Dyer A.J., Krause G.F., Hedrick H.B., 1973. Growth traits in straightbred and reciprocally crossed Angus, Hereford and Charolais steers. J. Anim. Sci., 36, 1044-1050.

Liboriussen T., Lauritzen F., Andersen B.B., Buchter L., Sorensen S.E., Klastrup S., KousGaARD K., 1982. Krydsnings-og produktionsforsog med europaeiske kodracer I og II (en Danois). Beret. fra Statens Husdyrbrugsfors., $527,65 \mathrm{p}$. 
LoNG C.R., 1980. Crossbreeding for beef production : experimental results. J. Anim. Sci., 51, 1197-1223.

LoNG C.R., Gregory K.E., 1975. Heterosis and management effects in postweaning growth of Angus, Hereford and reciprocal cross cattle. J. Anim. Sci., 41, 1563-1571.

Ménissier F., Sapa J., Foulley J.L., Frebling J., Bonaïti B., 1982. Comparison of different sire breeds crossed with Friesian cows : preliminary results. Curr. Top. Vet. Med. Anim. Sci., 21, 94-136.

Ménissier F., Bibé B., Perreau B., Vissac B., 1988. Comparaison des races bovines Charolaise, Limousine et Maine-Anjou en race pure et en intercroisement. 1. Objectif et dispositif expérimental. Génét. Sél. Evol., 20, 321-342.

Quesnel F., 1980. Analyse de la vitesse de croissance avant sevrage chez les vaches allaitantes. Mémoire Inst. Nat. Agronom. Paris-Grignon, $66 \mathrm{p}$.

Rahnefeld G.W., Fredeen H.T., Weiss G.M., Lawson J.E., Newman J.A., 1983. Breed of terminal sire effects on carcass characteristics of three-way cross beef cattle reared at two locations. Can. J. Anim. Sci., 63, 523-549.

Renand G., 1985. Genetic parameters of French beef breeds used in crossbreeding for young bull production. 1. Live performance. Génét. Sél. Evol., 17, 153-170.

Villareal E.L., 1985. Etude comparée des paramètres de la courbe de croissance de quatre races bovines à viande appartenant à des types biologiques différents. $150 \mathrm{p}$., Thèse d'Université, Université Paris-Sud. 